\title{
Quantitative assessment of cerebral metabolism and hemodynamics in small-for-gestational-age (SGA) newborns
}

\author{
Ying $\mathbf{Q i}^{1}$, Xiaoming Wang ${ }^{1}$, Jian Mao ${ }^{2}$ \\ ${ }^{1}$ Department of Radiology, Shenging Hospital of China Medical University, Shenyang, China; ${ }^{2}$ Department of Pediatrics, Shengjing Hospital of \\ China Medical University, Shenyang, China
}

Correspondence to: Dr. Ying Qi. Department of Radiology, Shengjing Hospital of China Medical University, No. 36 Sanhao Street, Heping District, Shenyang 110004, China. Email: womanman1982@163.com.

Background: Small-for-gestational-age (SGA) newborns represent approximately $10 \%$ of births worldwide and $45 \%$ of births in some countries. It has been suggested that SGA might cause learning difficulties and behavioral abnormalities in childhood, yet the neurobiological basis for this is poorly understood. In this study, we employed several advanced imaging techniques-including T2-relaxation-under-spin-tagging (TRUST) magnetic resonance imaging (MRI), and phase-contrast (PC) MRI — to quantify oxygen extraction fraction (OEF), global cerebral blood flow (CBF), and cerebral metabolic rate of oxygen $\left(\mathrm{CMRO}_{2}\right)$ to elucidate pathophysiological vulnerabilities of SGA neonates.

Methods: A total of 41 newborns were enrolled in this study, consisting of 29 SGA and 12 appropriatefor-gestational-age (AGA) neonates. The SGA group was further divided into subgroups with and without abnormalities on structural MRI, denoted as SGA-a $(\mathrm{N}=17)$ and SGA-n $(\mathrm{N}=12)$. TRUST and PC MRI were performed to determine $\mathrm{OEF}, \mathrm{CBF}$, and $\mathrm{CMRO}_{2}$. Linear regression analyses were performed to examine physiological parameters' dependence on scan age, gender, and group. Similar analyses were conducted for birth weight and brain volume. Receiver operating characteristic (ROC) curves were used to test physiological parameters' ability to different diagnostic groups.

Results: Regression analysis revealed that $\mathrm{CMRO}_{2}$ was significantly lower $(\mathrm{P}=0.04)$ in the SGA group relative to the AGA group. When further stratifying the SGA participants into SGA-a and SGA-n subgroups, the SGA-a subgroup was found to have the most pronounced physiological deficits, with a lower $\mathrm{CMRO}_{2}$ $(\mathrm{P}=0.004)$ and lower $\mathrm{CBF}(\mathrm{P}=0.007)$ than those in the AGA group. Conversely, $\mathrm{CMRO}_{2}(\mathrm{P}=0.40)$ and $\mathrm{CBF}$ $(\mathrm{P}=0.90)$ in the SGA-n subgroup were not different from those of the AGA group. Accordingly, CBF in the SGA-a group was significantly lower $(\mathrm{P}=0.01)$ than that of the SGA-n group and CMRO2 also showed a difference $(\mathrm{P}=0.09)$. Additionally, $\mathrm{CMRO}_{2}(\mathrm{P}=0.002)$ and $\mathrm{CBF}(\mathrm{P}=0.04)$ showed an age-related increase during this early developmental period. In analyzing the SGA-a subgroup relative to the remaining neonates, the area under curve (AUC) values were 0.6, 0.6, 0.7, 0.8, and 0.5 for birth weight, OEF, $\mathrm{CMRO}_{2}, \mathrm{CBF}$, and brain volume, respectively. In analyzing the SGA-a subgroup relative to the SGA-n subgroup, AUC values were 0.5, 0.6, 0.7, 0.8, and 0.5 for birth weight, $\mathrm{OEF}, \mathrm{CMRO}_{2}, \mathrm{CBF}$, and brain volume.

Conclusions: Structural damage in SGA-a neonates is associated with cerebral hemodynamic and metabolic deficits. SGA neonates with normal $\mathrm{CBF}$ and $\mathrm{CMRO}_{2}$ reveal minimal structural abnormalities. Physiological imaging may help identify SGA patients at high risk of developing irreversible brain damage.

Keywords: Small-for-gestational-age (SGA); T2-relaxation-under-spin-tagging (TRUST); phase-contrast (PC); magnetic resonance imaging (MRI); oxygen extraction fraction (OEF); oxygen saturation fraction in venous blood $(\mathrm{Yv})$; cerebral blood flow (CBF); cerebral metabolic rate of oxygen $\left(\mathrm{CMRO}_{2}\right)$

Submitted Sep 07, 2020. Accepted for publication Jan 07, 2021.

doi: $10.21037 /$ qims-20-1040

View this article at: http://dx.doi.org/10.21037/qims-20-1040 


\section{Introduction}

Small-for-gestational-age (SGA) newborns have birth weights and/or birth lengths greater than two standard deviations below established means or below the $10^{\text {th }}$ percentile. SGA and intrauterine growth restriction (IUGR) are often used interchangeably in the literature and clinical workflow (1). The global incidence of SGA newborns has gradually increased in recent years, with a current worldwide prevalence of $9.7 \%$ (2), with the highest percentage recorded in South Asia (45\%) (3). Compared with appropriate-for-gestational-age (AGA) newborns, intensive-care treatment, morbidity due to perinatal fetal distress and neonatal asphyxia, and perinatal mortality are more likely in SGA infants; they are also prone to hyperbilirubinemia, hypoglycemia, intracranial bleeding, anemia, and respiratory complications. SGA status is also known to influence the nervous system's development, resulting in learning difficulties and neurological/behavioral abnormalities in childhood $(4,5)$. Although most SGA infants show catch-up growth in height and weight, an increased risk of vascular diseases in adulthood has also been reported (6,7). Additionally, it has been suggested that catch-up or postnatal accelerated growth in low birthweight neonates may adversely affect cognitive function (8). Therefore, understanding neonatal brain function, such as cerebral oxygen metabolism and hemodynamics, may provide important pathophysiological insights in SGA infants.

Physiological and functional brain imaging in neonates is particularly challenging due to small brain size, susceptibility to motion, and limited scan time. Computed tomography (CT) (9), positron emission tomography (PET) (10), near-infrared spectroscopy (NIRS) $(11,12)$, and ${ }^{133}$ xenon clearance (13) are common methods of measuring cerebral blood flow (CBF) and metabolism in adults, but these procedures either involve radiation exposure, present difficulties with visualizing deep brain tissues, or require exogenous tracers; thus, their application in neonatal brain imaging has been limited. Recent advances in magnetic resonance imaging (MRI) technologies have allowed non-invasive and quantitative measurement of brain hemodynamics and metabolism without the need for contrast agents. The brain's oxygen extraction fraction (OEF) can be determined using a T2-relaxation-underspin-tagging (TRUST) MRI technique (14), while global $\mathrm{CBF}$ can be measured using quantitative flow phasecontrast (PC) MRI. Therefore, OEF and CBF can be combined to assess the global cerebral metabolic rate of oxygen $\left(\mathrm{CMRO}_{2}\right)(14)$. Importantly, the entirety of these physiological measurements can be completed in less than 5 minutes, making their application practical in the neonatal population $(15,16)$. It should also be noted that arterial spin labeling (ASL), another important perfusion MRI technique, was considered but tends to have a lower signalto-noise ratio, longer scan time, and greater susceptibility to confounding factors such as the bolus arrival time.

In previous research, infants with hypoxic-ischemic encephalopathy (HIE) had lower $\mathrm{CBF}$ and $\mathrm{CMRO}_{2}$ using the $\mathrm{T} 2$ prepared tissue relaxation inversion recovery (T2TRIR) pulse sequence and ASL (17). Newborns with severe HIE had lower CBF and extracted less oxygen than those with moderate HIE using ASL and NIRS. A strong correlation was found between $\mathrm{CMRO}_{2}$ and $\mathrm{CBF}$ in asphyxiated newborns with severe HIE ( $\mathrm{r}=0.88)$ (18). Our previous work found newborns with white matter damage had lower oxygen consumption and CBF on PC MRI and TRUST MRI (15). Baenziger reported that CBF in SGA neonates was significantly higher than in AGA neonates $(16.1 \pm 4.1$ vs. $11.5 \pm 2.6 \mathrm{~mL} / 100 \mathrm{~g} / \mathrm{min})$ using ${ }^{133}$ xenon clearance (19). Few perinatal studies of cerebral oxygen metabolism and hemodynamics in SGA, especially grouping based on abnormal structure, have been reported.

In the present study, we used TRUST MRI and PC MRI to determine OEF, CBF, and $\mathrm{CMRO}_{2}$ in a group of SGA newborns and compared them to a group of AGA neonates.

\section{Methods}

\section{Participants}

The Ethics Committee approved the study protocol of Shengjing Hospital of China Medical University (IRB2015PS28K). Written informed consent was waived because the data were collected as part of a clinically indicated MRI. Between July 2016 and December 2017, a total of 41 newborns were enrolled. Structural MRI pulse sequences were performed, including axial and sagittal $T_{1-}$ weighted MRI, axial $\mathrm{T}_{2}$-weighted MRI, and diffusionweighted imaging (DWI). The exclusion criteria were congenital malformations, severe infection, or unusable MRI images. The participant was categorized as SGA if their birth weight was less than the $10^{\text {th }}$ percentile for gestational age, according to the 2013 Fenton \& Kim growth chart (20). The AGA participants were required to meet the birth weight criteria greater than the $10^{\text {th }}$ 
percentile and the absence of structural MRI abnormalities. The participants were further divided into those with abnormal MRI (SGA-a) within the SGA group and those with normal MRI (SGA-n). Grouping of SGA neonates with or without brain injury was determined by the pediatrician based on MRI. There is often no difference between SGA and AGA infants' neurobehavioral development during the perinatal period (21); MRI is often used to observe neonatal brain damage and help determine injury type, injury severity, gray and white matter volume, and prognosis. Imaging helps pediatricians make an accurate diagnosis, prescribe helpful and safe treatment, and develop long-term follow-up observations based on SGA grouping. Rating of MRI for abnormalities was conducted by an experienced neuroradiologist (Y.Q., with 9 years of experience).

\section{General MRI protocol}

A 3T MRI system equipped with a phased array head coil (Intera Achieva; Philips Healthcare, Best, Netherlands) was used for all MRI studies. Before imaging, pediatrician sedated infants using chloral hydrate nasal feeding $(50 \mathrm{mg} / \mathrm{kg})$ following the standard clinical practice guidelines at our institution. All newborns were well-fed, visually monitored, and fitted with hearing protection during the scans.

The axial $\mathrm{T}_{1}$-weighted spin-echo images were acquired with the following parameters: repetition time/echo time (TR/TE), $200 \mathrm{~ms} / 2.3 \mathrm{~ms}$; section thickness, $5 \mathrm{~mm}$; the field of view (FOV), $180 \times 150 \times 89 \mathrm{~mm}^{3}$; matrix size, $224 \times 162$; and scan time, $34.4 \mathrm{~s}$. Sagittal $\mathrm{T}_{1}$-weighted spin-echo images used the following parameters: TR/TE, $250 \mathrm{~ms} / 2.3 \mathrm{~ms}$; section thickness, $5 \mathrm{~mm}$; FOV, $230 \times 230 \times 107 \mathrm{~mm}^{3}$; matrix size, $256 \times 250$; and scan time, $33.0 \mathrm{~s}$. Axial $\mathrm{T}_{2}$-weighted imaging used the following parameters: TR/TE, 5,000 ms/ $80 \mathrm{~ms}$; section thickness, $5 \mathrm{~mm}$; FOV, $180 \times 150 \times 90 \mathrm{~mm}^{3}$; matrix size, $112 \times 112$; and scan time, 40.9 s. Axial echoplanar imaging (EPI) DWI was performed as follows: TR/TE, 3,500 ms/63 ms; section thickness, $5 \mathrm{~mm}$; FOV, $180 \times 180 \times 89 \mathrm{~mm}^{3}$; b values 0 and $1,000 \mathrm{~s} / \mathrm{mm}^{2}$; and scan time, $30.0 \mathrm{~s}$.

\section{Measurement of $O E F$}

OEF can be determined by:

$$
\mathrm{OEF}=\left(\mathrm{Y}_{\mathrm{a}}-\mathrm{Y}_{\mathrm{v}}\right) / \mathrm{Y}_{\mathrm{a}} \times 100 \%
$$

where $Y_{a}$ and $Y_{v}$ represent arterial and venous oxygenation, respectively. $\mathrm{Y}_{\mathrm{a}}$ was measured peripherally using a pulse oximeter on the toe. $Y_{v}$ was measured using a TRUST MRI technique. TRUST MRI is based on the principle that blood $T_{2}$ has a calibratable relationship with oxygenation level; the sequence applies a spin labeling module to isolate pure venous blood signals, then employs a series of $\mathrm{T}_{2}$-preparation pulses to modulate the MRI signal, the mono-exponential fitting of which yields blood $T_{2}$. A hematocrit (Hct)-specific $T_{2}-Y_{v}$ calibration curve was used to convert venous $T_{2}$ to oxygenation, where by Hct was based on newborns' blood test results (22). The imaging slice of TRUST MRI was placed according to previously established procedures $(14,15)$, parallel to the intercommissural lines with a $10-\mathrm{mm}$ distance from the sinus confluence [postmenstrual age $(\mathrm{PMA})>36$ weeks] or directly through sinus confluence (PMA $<36$ weeks) (Figure 1). Other parameters included: slab thickness, $80 \mathrm{~mm}$; four effective TEs (eTE) of $0,40,80$, and $160 \mathrm{~ms}$; TR, $3000 \mathrm{~ms}$; inversion time (TI), 1,022 ms; FOV, $160 \times 160 \times 5 \mathrm{~mm}^{3}$; matrix size, $64 \times 61$; SENSE factor, 3 ; voxel size, $2.5 \times 2.5 \times 5 \mathrm{~mm}^{3}$; $\tau$ CPMG, $10 \mathrm{~ms}$; eTEs, three pairs; and total scan time, $72 \mathrm{~s}$. Data processing of TRUST MRI followed previously established procedures (14-16). Global $Y_{v}$ was combined with $Y_{a}$ to provide an OEF estimation.

\section{Measurement of CBF}

Quantitative flow PC MRI was used to measure CBF. PC MRI employs bipolar gradients to encode flow velocity of blood in major arteries of the brain, specifically the left internal carotid artery (LICA), right internal carotid artery (RICA), left vertebral artery (LVA), and right vertebral artery (RVA), providing a measurement of total blood flow to the brain. The flow value can then be normalized using brain volume to obtain $\mathrm{CBF}$ in the units of $\mathrm{mL} / 100 \mathrm{~g} / \mathrm{min}$. A time-of-flight (TOF) MR angiogram (MRA) was first performed to allow visualization of the arteries mentioned above (Figure 1). The TOF-MRA was conducted by positioning imaging slabs at the center of the epistropheus and stationing $60-\mathrm{mm}$ saturation slabs above to suppress venous signals. The settings of TOF-MRA were: TR/TE, $20 \mathrm{~ms} / 3.45 \mathrm{~ms}$; flip angle, $18^{\circ}$; FOV, $90 \times 90 \times 20 \mathrm{~mm}^{3}$; voxel size, $0.8 \times 0.8 \times 2 \mathrm{~mm}^{3}$; and scan duration, $23.7 \mathrm{~s}$.

Based on the TOF-MRA images, four PC MRI scans were performed by placing the imaging slice perpendicular and centered on each target artery, respectively, according to previous procedures $(14,15)$ (Figure 1). Those of the LICA and RICA was placed at the foramen magnum level, whereas the LVA and RVA imaging slices were placed 

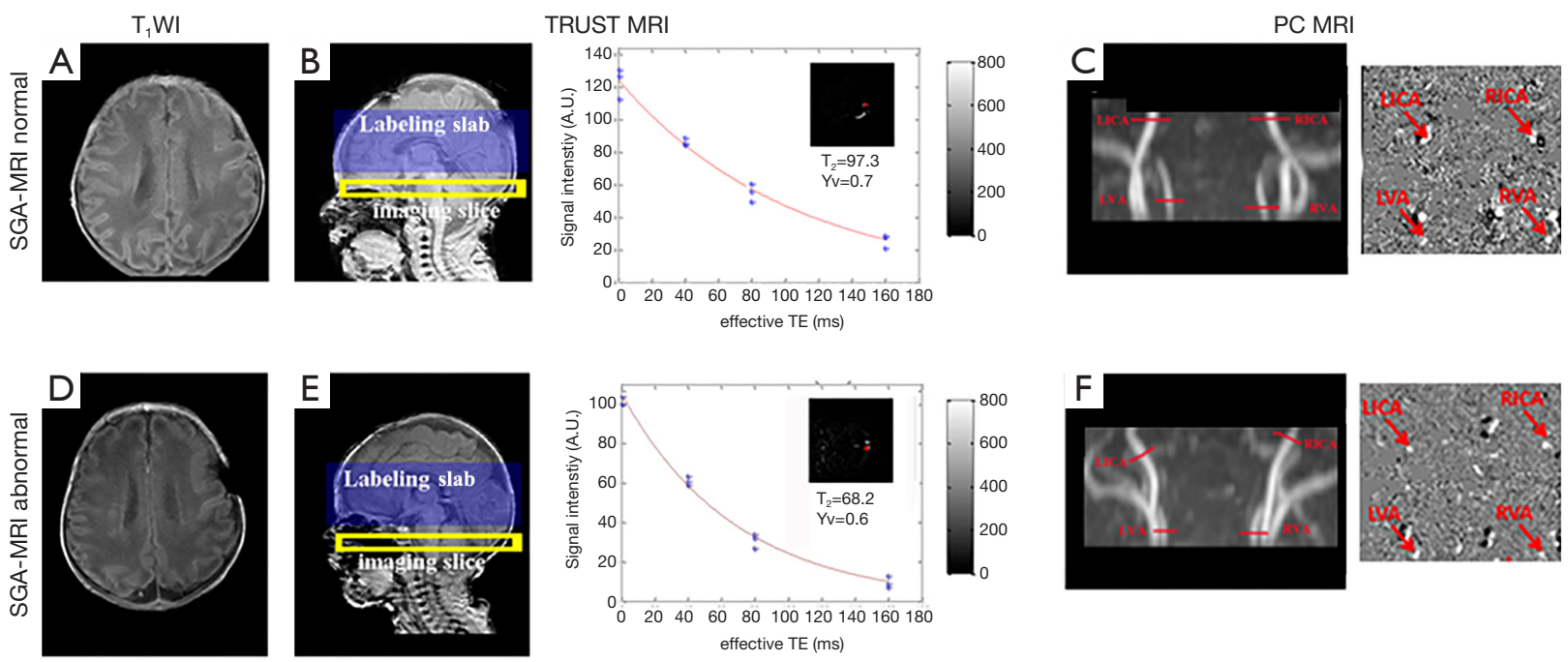

Figure 1 Measurement of $\mathrm{Yv}_{v}$ and CBF using T2-relaxation-under-spin-tagging (TRUST) and phase-contrast (PC) MRI. (A,D) Axial T1weighted imaging ( $\left.\mathrm{T}_{1} \mathrm{WI}\right)$; (B,E) sagittal $\mathrm{T}_{1} \mathrm{WI}$ for positioning of TRUSTMRI study; views at effective TEs (eTEs) of 0, 40, 80, and 160 ms; mono-exponential fitting of signal intensity in targeted venous sinus (as function of e'TE) yielding venous blood, $\mathrm{T}_{2}$ then converted to $\mathrm{Yv}_{\mathrm{v}}$ via calibration plot; (C,F) positioning for PCMRI of internal carotid arteries (left, LICA; right, RICA) and vertebral arteries (left, LVA; right, RVA), using maximal-intensity-projection (MIP) angiogram images; velocity maps of LICA, RICA, LVA, and RVA. (A,B,C) show 35.7-old newborn (birth weight, 1,780 g) in SGA-n group. There was normal signal intensity on $\mathrm{T}_{1} \mathrm{WI}$; (D,E,F) as above of 35.1 week-old newborn (birth weight, 1,200 g) in SGA-a group. There were multiple areas of 35.7-week white matter damage enclosed in the periventricular areas. LICA, left internal carotid artery; RICA, right internal carotid artery; SGA, small-for-gestational-age.

immediately below epistropheus, away from the pivot points. Imaging parameters were: single slice; voxel size, $0.5 \times 0.5 \times 3 \mathrm{~mm}^{3}$; FOV, $180 \times 180 \times 3 \mathrm{~mm}^{3}$; maximum velocity encoding, $20 \mathrm{~cm} / \mathrm{s}$ (non-gated); averages, 2; total scan (four arteries), $1.5 \mathrm{~min}$.

Data processing of PC MRI followed the previously described procedures $(14,16)$. Manual delineation of the LICA, RICA, LVA, and RVA was conducted based on each PC MRI's magnitude image, respectively, and the ROI masks were applied to the velocity maps to obtain flux in these vessels. The sum of fluxes in all vessels yielded the whole brain's blood flow rate $(\mathrm{mL} / \mathrm{min})$. To convert the value to $\mathrm{CBF}$, brain volume was obtained by manually tracing the $T_{2}$-weighted images and assuming a parenchymal density of $1.06 \mathrm{~g} / \mathrm{mL}(23)$. CBF $(\mathrm{mL} / 100 \mathrm{~g} / \mathrm{min})$ was then calculated by dividing the total flux by brain weight.

\section{Calculation of $\mathrm{CMRO}_{2}$}

Once $\mathrm{OEF}$ and $\mathrm{CBF}$ were obtained, $\mathrm{CMRO}_{2}$ was calculated based on Fick's principle (24,25):

$$
\mathrm{CMRO}_{2}=\mathrm{CBF} \times \mathrm{OEF} \times \mathrm{Y}_{\mathrm{a}} \times \mathrm{C}_{\mathrm{a}}
$$

where the unit of $\mathrm{CMRO}_{2}$ is $\mu \mathrm{mol} \mathrm{O}_{2} / 100 \mathrm{~g} / \mathrm{min}$. $\mathrm{C}_{\mathrm{a}}$ represents the oxygen-carrying capacity of blood and is assumed to be $897 \mu \mathrm{mol} \mathrm{O}_{2} / 100 \mathrm{~mL}$ for a Hct of $44 \%$ (26). Each neonate used a subject-specific $\mathrm{C}_{\mathrm{a}}$ based on their Hct level.

\section{Statistical analysis}

Regression analysis was used to analyze the scan age and gender differences between SGA and AGA groups, SGA-a and SGA-n groups, SGA-a and AGA groups, and SGA-n and AGA groups, respectively, treating the groups as 0 and 1 , and using scan age and gender as covariates. A linear regression analysis was used to examine physiological parameters' dependence on scan age, gender, and diagnostic group; the physiological parameter was assigned as the dependent variable, and scan age, gender, and the group were assigned as independent variables. The following coding was used in the regression analyses between pairs of groups: SGA group and AGA group were coded as 0 and 1 , respectively; SGA-a and SGA-n as 1 and 0; SGA-a and 
Table 1 Clinical characteristics of the 41 infants and their OEF, $\mathrm{CMRO}_{2}, \mathrm{CBF}$ and brain volume in SGA (containing SGA-MRI abnormal and SGA-MRI normal) and AGA groups

\begin{tabular}{|c|c|c|c|c|c|c|c|c|}
\hline Group & $\mathrm{N}$ & $\begin{array}{c}\text { Scan age } \\
\text { (weeks) }\end{array}$ & $\begin{array}{c}\text { Birth age } \\
\text { (weeks) }\end{array}$ & Birth weight (g) & OEF (\%) & $\begin{array}{c}\mathrm{CMRO}_{2} \\
(\mu \mathrm{mol} / 100 \mathrm{~g} / \mathrm{min})\end{array}$ & $\begin{array}{c}\text { CBF } \\
(\mathrm{mL} / 100 \mathrm{~g} / \mathrm{min})\end{array}$ & $\begin{array}{c}\text { Brain volume } \\
(\mathrm{mL})\end{array}$ \\
\hline SGA & 29 & $37.5 \pm 3.2$ & $33.5 \pm 4.2$ & $1,811.5 \pm 612.2$ & $37.0 \pm 14.4$ & $33.6 \pm 12.2$ & $13.8 \pm 5.3$ & $304.0 \pm 82.7$ \\
\hline SGA-n & 12 & $37.4 \pm 3.6$ & $33.3 \pm 4.0$ & $1,815.9 \pm 666.7$ & $34.9 \pm 9.1$ & $38.4 \pm 13.3$ & $17.0 \pm 4.7$ & $311.2 \pm 64.2$ \\
\hline AGA & 12 & $36.6 \pm 3.7$ & $35.5 \pm 3.9$ & $2,777.5 \pm 1,117.1$ & $31.4 \pm 4.8$ & $40.1 \pm 11.4$ & $16.0 \pm 3.8$ & $311.5 \pm 81.1$ \\
\hline
\end{tabular}

Parameter values are reported as mean \pm standard deviation. SGA, small-for-gestational-age; SGA-a, SGA neonates with abnormal structural MRI; SGA-n, SGA neonates with normal structural MRI.

AGA as 1 and 0; and SGA-n and AGA as 0 and 1. If scan age or gender was related to $\mathrm{CMRO}_{2}, \mathrm{CBF}$, or $\mathrm{OEF}$, it was necessary to correct birth weight, $\mathrm{CMRO}_{2}, \mathrm{CBF}, \mathrm{OEF}$, and brain volume for age and gender before group comparison. For example, corrected OEF was calculated using the formula:

$$
\begin{aligned}
\text { Corrected } \mathrm{OEF}= & \text { OEF- } \mathrm{b}_{1} \times(\text { age }-\overline{\mathrm{age}})-\mathrm{b}_{2} \\
& \times(\text { gender }- \text {-gender })
\end{aligned}
$$

The coefficients $b_{1}$ and $b_{2}$ were based on the scan age and gender regression model results between SGA and AGA groups, SGA-a and SGA-n groups, SGA-a and AGA groups, and SGA-n and AGA groups, respectively, where similar statistical analysis had been reported (27). Corrected birth weight, brain volume, and other physiological parameters were compared using an independent-samples $t$-test between SGA and AGA, SGA-a and SGA-n, SGA-a and AGA, and SGA-n and AGA groups, separately. Birth weight, brain volume, and cerebral physiological parameters also independently underwent receiver operating characteristic (ROC) curve analysis to distinguish SGA-a from the remaining neonates. Birth weight, brain volume, and cerebral physiological parameters also independently underwent ROC curve analysis to distinguish SGA-a from SGA-n neonates. These statistical analyses used the software SPSS (v21; IBM Corp, Armonk, NY, USA). A P value of 0.05 or less was considered statistically significant.

\section{Results}

\section{Participant characteristics}

Demographic and clinical characteristics of group participants and their $\mathrm{OEF}, \mathrm{CMRO}_{2}, \mathrm{CBF}$, and brain volumes are summarized in Table 1. The SGA-n group included 12 neonates (male, 11; female, 1). The SGA-a group included 17 neonates (male, 10; female, 7); five displayed hypoxic/ischemic brain injuries, and five had suffered from premature rupture of membranes (PROM). Their MRI abnormalities included: eight cystic lesions, five instances of white-matter damage, three subarachnoid hemorrhages, and one cerebral infarction (Figure 2). The SGA group included the sum of SGA-n and SGA-a participants, thus consisted of 29 subjects in total. The AGA group included 12 neonates (male, 9; female, 3). These subjects received clinically indicated MRI due to certain clinical signs/symptoms, umbilical cord blood gas analysis, or other routine blood tests at birth; however, no MRI abnormalities were observed, presumably excluding any nervous system disease.

\section{Dependence of cerebral hemodynamics and metabolism on diagnostic groups}

Figure 1 shows TRUST and PC MRI data from two representative subjects. We first compared the physiological measures of SGA and AGA neonates (Table 2). $\mathrm{CMRO}_{2}$ was significantly lower $(\mathrm{P}=0.04)$ in the SGA group relative to the AGA group, but there was no significant difference in $\mathrm{CBF}$ $(\mathrm{P}=0.12)$ or $\mathrm{OEF}(\mathrm{P}=0.21)$. Additionally, $\mathrm{CMRO}_{2}(\mathrm{P}=0.002)$ and $\mathrm{CBF}(\mathrm{P}=0.04)$ showed an age-related scan increase during this early developmental period. Birth weight, but not brain volume, was significantly different between the SGA and AGA neonates.

Next, we divided the SGA neonates into SGA-a and SGA-n subgroups. Compared to the AGA neonates, the SGA-a group had a lower $\mathrm{CMRO}_{2}(\mathrm{P}=0.004)$ and lowered CBF $(\mathrm{P}=0.007)$ than the AGA group. Conversely, $\mathrm{CMRO}_{2}$ $(\mathrm{P}=0.40)$ and $\mathrm{CBF}(\mathrm{P}=0.90)$ in the SGA-n group were not different from the AGA group. Accordingly, CBF in the 

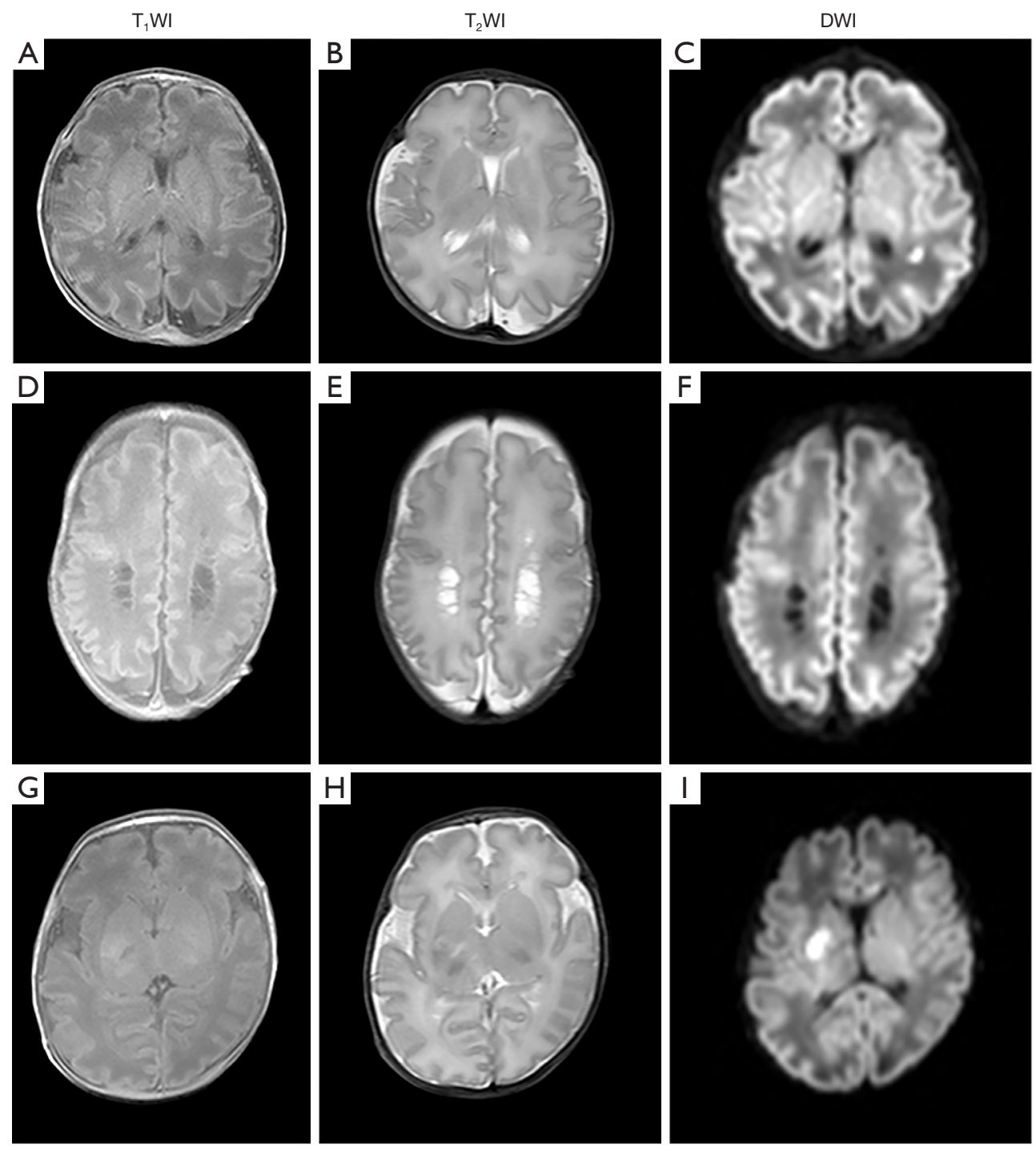

Figure $2 \mathrm{~T}_{1}$ WI, $\mathrm{T}_{2} \mathrm{WI}$ and DWI for SGA-a neonates. (A,B,C) A 34.4-week-old newborn (birth weight, 1,840 g; male) with white matter damage and increased signal intensity on $\mathrm{T}_{1} \mathrm{WI}$ and DWI; (D,E,F) a 36.1-week-old newborn (birth weight, 1,125 g; male) with periventricular leukomalacia with multiple cystic lesions enclosed in bilateral centrum semiovale. (G,H,I) A 36.1-week-old newborn (birth weight, 1,140 g; male) with cerebral infarction with mixed signal intensity in the right basal ganglia on $\mathrm{T}_{1} \mathrm{WI}$, and increased signal intensity on $\mathrm{T}_{2} \mathrm{WI}$ and DWI. $\mathrm{T}_{1}$ WI, T1-weighted imaging; DWI, diffusion-weighted imaging; SGA, small-for-gestational-age.

SGA-a group was significantly lower $(\mathrm{P}=0.01)$ than that in the SGA-n group, and $\mathrm{CMRO}_{2}$ also showed a trend of difference $(\mathrm{P}=0.09)$ between the SGA-a and SGA-n groups. OEF results showed no statistically significant differences between SGA and AGA groups $(\mathrm{P}=0.21), \mathrm{SGA}-\mathrm{a}$ and SGA-n groups $(\mathrm{P}=0.52), \mathrm{SGA}-\mathrm{a}$ and AGA groups $(\mathrm{P}=0.19)$, and SGA-n and AGA groups $(\mathrm{P}=0.35)$, separately.

Regression analysis showed no significant differences in scan age and gender between SGA and AGA groups $(\mathrm{P}=0.45$,
$\mathrm{P}=0.99)$, SGA-a and SGA-n groups $(\mathrm{P}=0.95, \mathrm{P}=0.08)$, SGA-a, and AGA groups $(\mathrm{P}=0.58, \mathrm{P}=0.48)$, and SGA-n and AGA groups $(\mathrm{P}=0.48, \mathrm{P}=0.26)$, respectively.

Table 2 shows detailed results of coefficients (scan age: $b_{1}$; gender: $b_{2}$ ), $95 \%$ confidence interval (CI) of coefficients, and their $\mathrm{P}$ values. Group comparisons of corrected birth weight, $\mathrm{CMRO}_{2}, \mathrm{CBF}, \mathrm{OEF}$, and brain volume for scan age and gender had similar results. The corrected $\mathrm{CMRO}_{2}$ and corrected $\mathrm{CBF}$ showed significant differences between 


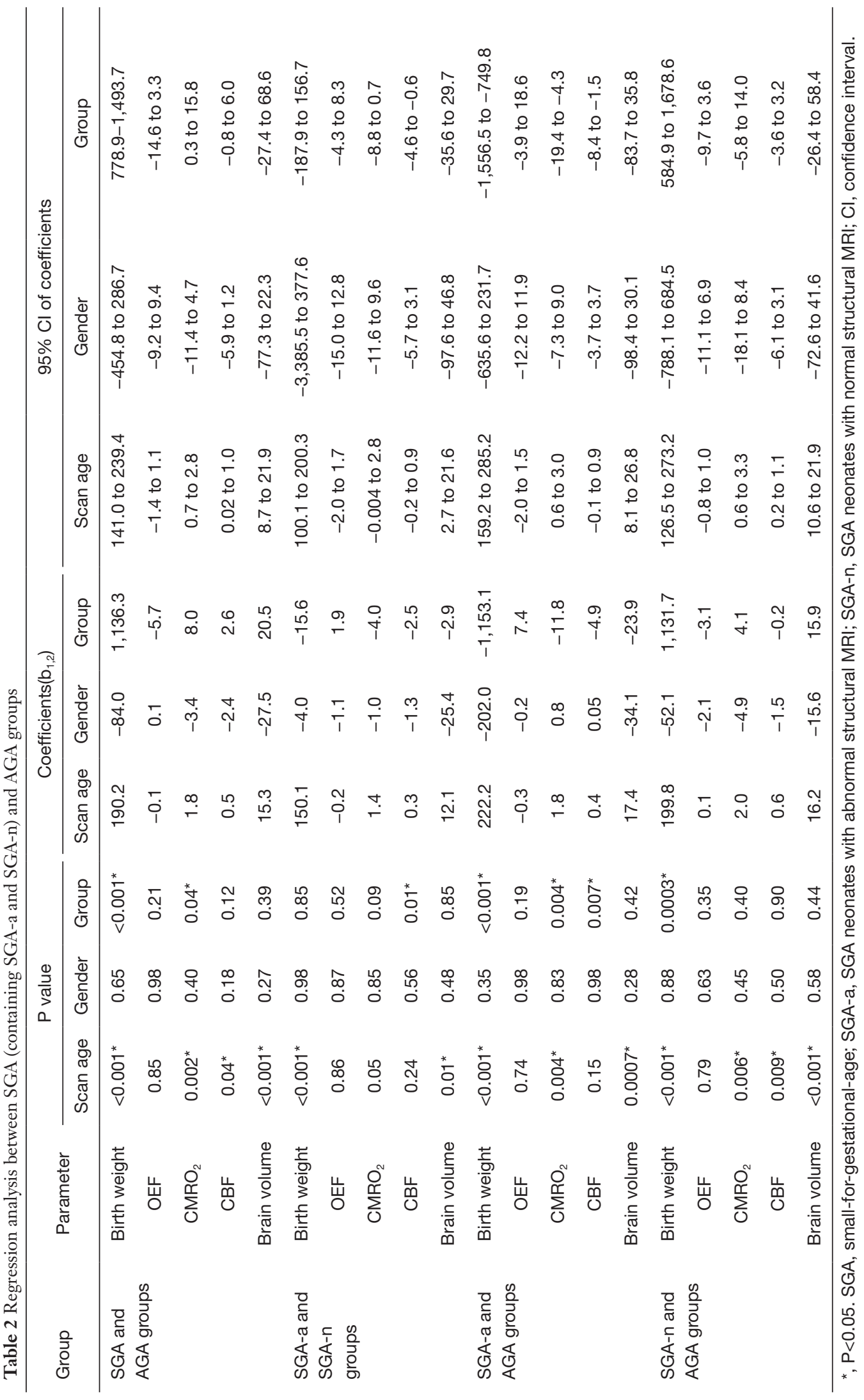


Table 3 Comparison of corrected values between groups and their subgroups

\begin{tabular}{|c|c|c|c|c|c|}
\hline Group & Corrected birth weight (g) & Corrected OEF (\%) & $\begin{array}{l}\text { Corrected } \mathrm{CMRO}_{2} \\
\text { ( } \mu \mathrm{mol} / 100 \mathrm{~g} / \mathrm{min})\end{array}$ & $\begin{array}{l}\text { Corrected CBF } \\
(\mathrm{mL} / 100 \mathrm{~g} / \mathrm{min})\end{array}$ & $\begin{array}{l}\text { Corrected brain volume } \\
\qquad(\mathrm{mL})\end{array}$ \\
\hline SGA & $1,761.7 \pm 404.9$ & $37.0 \pm 14.1$ & $33.1 \pm 11.4$ & $13.7 \pm 5.1$ & $300.2 \pm 73.4$ \\
\hline AGA & $2,897.7 \pm 636.8$ & $31.3 \pm 4.6$ & $41.1 \pm 8.0$ & $16.3 \pm 3.1$ & $320.7 \pm 37.9$ \\
\hline $\mathrm{t}$ & -6.6 & 1.9 & -2.2 & -1.6 & -0.9 \\
\hline SGA-n & $1,829.8 \pm 445.9$ & $34.7 \pm 8.7$ & $38.3 \pm 12.1$ & $16.8 \pm 4.2$ & $307.5 \pm 48.0$ \\
\hline $\mathrm{t}$ & -0.2 & 0.7 & -1.9 & -3.0 & -0.2 \\
\hline $\mathrm{P}$ & 0.80 & 0.48 & 0.06 & $0.01^{*}$ & 0.84 \\
\hline $\mathrm{P}$ & $<0.001^{*}$ & 0.13 & $0.004^{*}$ & $0.007^{*}$ & 0.33 \\
\hline SGA-n & $1,731.1 \pm 520.9$ & $34.7 \pm 9.0$ & $37.2 \pm 12.5$ & $16.6 \pm 4.3$ & $303.4 \pm 52.5$ \\
\hline AGA & $2,862.8 \pm 652.4$ & $31.6 \pm 4.8$ & $41.3 \pm 8.3$ & $16.4 \pm 3.0$ & $319.3 \pm 37.9$ \\
\hline $\mathrm{t}$ & -4.7 & 1.0 & -0.9 & 0.1 & -0.9 \\
\hline$P$ & $0.0003^{*}$ & 0.31 & 0.40 & 0.90 & 0.40 \\
\hline
\end{tabular}

Parameter values are reported as mean \pm standard deviation. * $\mathrm{P}<0.05$. SGA, small-for-gestational-age; SGA-a, SGA neonates with abnormal structural MRI; SGA-n, SGA neonates with normal structural MRI.

SGA-a and AGA groups $(\mathrm{P}=0.004, \mathrm{P}=0.007)$. This suggested that the differences in physiological parameters $\left(\mathrm{CMRO}_{2}\right.$ and CBF) between SGA-a and AGA groups were related to brain injury, not scan age (Table 3$)$.

\section{ROC curves in identifying SGA with abnormal structural MRI}

Figure $3 A$ shows ROC curves for birth weight [area under the curve $(\mathrm{AUC})=0.6,95 \%$ confidence interval $(\mathrm{CI})$ : 0.5-0.8), OEF (AUC =0.6, 95\% CI: 0.4-0.8), $\mathrm{CMRO}_{2}$ $(\mathrm{AUC}=0.7,95 \% \mathrm{CI}: 0.5-0.9), \mathrm{CBF}(\mathrm{AUC}=0.8,95 \% \mathrm{CI}$ : $0.6-0.9$ ), and brain volume (AUC $=0.5,95 \% \mathrm{CI}: 0.3-0.7$ ) used to distinguish SGA-a neonates from AGA and SGA-n neonates. Figure $3 B$ shows ROC curves of birth weight (AUC $=0.5,95 \%$ CI: 0.3-0.7), OEF (AUC $=0.6,95 \%$ CI: $0.2-0.7), \mathrm{CMRO}_{2}(\mathrm{AUC}=0.7,95 \% \mathrm{CI}: 0.5-0.9), \mathrm{CBF}$ (AUC $=0.8,95 \%$ CI: $0.6-1.0)$, and brain volume $(\mathrm{AUC}=0.5$, 95\% CI: $0.3-0.8$ ) used to distinguish SGA-a neonates from SGA-n neonates. The AUC values for CBF and $\mathrm{CMRO}_{2}$ all indicated fair diagnostic utility for identifying SGA-a. It was also observed that physiological parameters yielded higher AUC values than brain volume or body weight.

\section{Discussion}

To our knowledge, the present study is the first to determine oxygen consumption and CBF in SGA neonates. Our data suggest that, while SGA newborns generally have lower metabolism and CBF, patients with structural abnormalities show particularly pronounced deficits in these physiological parameters. $\mathrm{CMRO}_{2}$ and $\mathrm{CBF}$ provide a greater ability to identify SGA neonates with abnormal structural MRI, compared with existing birth weight or brain volume measures.

\section{Physiological and clinical considerations}

This study's major finding was that SGA neonates with normal structural MRI and AGA neonates have similar $\mathrm{CMRO}_{2}$ and $\mathrm{CBF}$; however, $\mathrm{CMRO}_{2}$ and $\mathrm{CBF}$ were lower in SGA neonates with abnormal structural MRI. CBF augmentation in SGA infants with normal structural MRI 

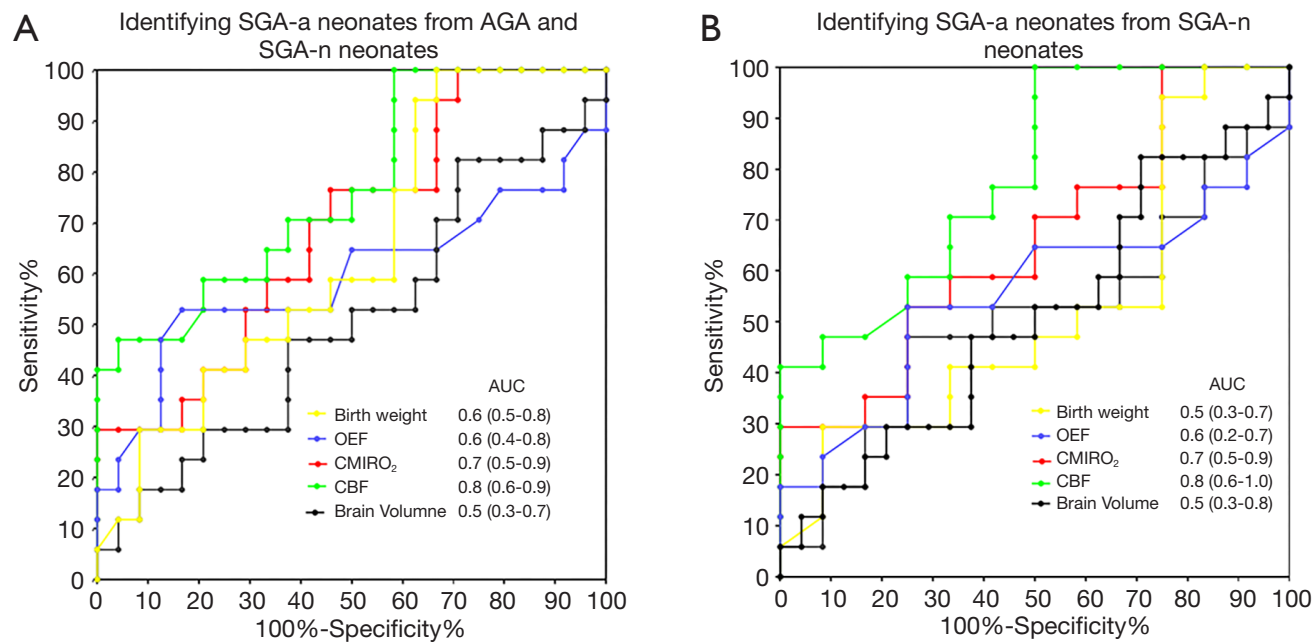

Figure 3 Comparison of ROC curves for five models (birth weight, OEF, $\mathrm{CMRO}_{2}, \mathrm{CBF}$, brain volume) to identify SGA newborns with abnormal structural MRI (denoted SGA-a). (A) ROC curves used to distinguish SGA-a neonates from AGA and SGA newborns with normal structural MRI (denoted SGA-n). (B) ROC curves used to distinguish SGA-a neonates from SGA-n neonates. CBF and CMRO ${ }_{2}$ all showed fair utility in diagnosing SGA-a. The physiological parameters had higher AUC than the morphological index of brain volume. ROC, receiver operating characteristic; SGA, small-for-gestational-age; OEF, oxygen extraction fraction; $\mathrm{CMRO}_{2}$, cerebral metabolic rate of oxygen; CBF, cerebral blood flow.

may correspond with attempted catch-up growth. Perinatal and early postpartum periods are critical developmental phases for low-birthweight neonates (8). In preterm infants or fetuses, growing tissues are characterized by high plasticity and high cellular proliferation rates. Conversely, such processes may alter biochemical and hormonal pathways, increase disease susceptibility in later life, incur long-term neurological deficits, and adversely impact cognitive function $(8,25)$.

Furthermore, premature and low-birthweight newborns have immature cerebrovascular systems. Once disruption to oxygen supply and metabolism occurs, $\mathrm{CMRO}_{2}$ may decline accordingly, and the likelihood of brain injury is increased due to poor cerebrovascular function. These notions were supported by the significant differences in $\mathrm{CMRO}_{2}$ and $\mathrm{CBF}$ observed between SGA neonates with abnormal structural MRI and AGA neonates. Thus, the hemodynamic and metabolic function may play a critical role in determining long-term, irreversible brain injury in SGA neonates.

\section{Technical considerations}

PC MRI and oxygenation MRI have previously been used to study OEF, $\mathrm{CMRO}_{2}$, and $\mathrm{CBF}$ in healthy and hypoxic-ischemic encephalopathic (HIE) neonates $(14,16)$
(Table 4). The physiological parameters measured in the AGA and SGA-n groups were similar to those reported in healthy infants (14), although CBF values appeared slightly higher in our study population $(16.0 \pm 3.8$ vs. $13.4 \pm 4.2$ $\mathrm{mL} / 100 \mathrm{~g} / \mathrm{min}$ ); differences in scan age, birth age, and birth weight may have contributed to some of the differences. Compared with the SGA-n group, SGA-a neonates had lower $\mathrm{CMRO}_{2}$ values, which was consistent with results previously reported in HIE neonates (16); however, CBF values in our study population were lower than those in HIE neonates $(11.5 \pm 4.5$ vs. $17.5 \pm 6.3 \mathrm{~mL} / 100 \mathrm{~g} / \mathrm{min})$. Aside from age, gender, and weight effects, the higher CBF reported in HIE neonates might also be related to therapeutic hypothermia (16). These findings support TRUST MRI and PC MRI's accuracy and reliability in assessing cerebral hemodynamics and metabolism of newborns.

\section{Relationship between physiological parameters and age}

We found that $\mathrm{CMRO}_{2}$ and CBF increased with age, which was consistent with previous reports $(14,15)$ and may reflect the increased energy demands of an important developmental period. Therefore, cerebral blood supply and oxygen metabolism appear to increase to match brain development's rapid rate. 
Table 4 Comparison of physiological parameters vs. previously published data

\begin{tabular}{|c|c|c|c|c|c|c|c|}
\hline Study & Method & Number of subjects and condition & $\begin{array}{l}\text { Scan age } \\
\text { (weeks) }\end{array}$ & Ya (\%) & OEF (\%) & $\begin{array}{c}\mathrm{CMRO}_{2}(\mu \mathrm{mol} / 100 \\
\mathrm{g} / \mathrm{min})\end{array}$ & $\begin{array}{c}\text { CBF } \\
(\mathrm{mL} / 100 \mathrm{~g} / \mathrm{min})\end{array}$ \\
\hline Shetty (16) & PC and TRUST & 5 hypoxic ischemic encephalopathy & $39 \pm 1.4$ & $96.8 \pm 2.6$ & $24.4 \pm 5$ & $34.74 \pm 8.3$ & $17.5 \pm 6.3$ \\
\hline This study & PC and TRUST & 12 SGA-n & $37.4 \pm 3.6$ & $95.0 \pm 2.0$ & $34.9 \pm 9.1$ & $38.4 \pm 13.3$ & $17.0 \pm 4.7$ \\
\hline
\end{tabular}

Parameter values are reported as mean \pm standard deviation. SGA, small-for-gestational-age; SGA-a, SGA neonates with abnormal structural MRI; SGA-n, SGA neonates with normal structural MRI; TRUST, T2-relaxation-under-spin-tagging; PC, phase-contrast.

Regression analysis showed no significant differences in scan age and gender between groups $(\mathrm{P}>0.05)$, suggesting that $\mathrm{CMRO}_{2}$ and CBF's significant differences between groups were not related to scanning age or gender. Table 3 confirmed this, showing similar differences between groups for corrected $\mathrm{CMRO}_{2}$ and $\mathrm{CBF}$.

\section{AUC values of physiological parameters used in identifying $S G A-a$ neonates}

We observed that physiological parameters had higher AUC values than the morphological index of brain volume. Although $T_{1}$ - and $T_{2}$-weighted scans readily identified structural abnormalities in SGA neonates, they could not provide information regarding these abnormalities' etiologies. The data showed that SGA-a neonates had lower CBF compared with SGA-n and AGA groups. Perinatal declines in cerebral blood supply and oxygen metabolism may trigger pathophysiological responses with longterm, irreversible consequences, preceding morphological changes. Our ROC curve suggests that $\mathrm{CBF}$ and $\mathrm{CMRO}_{2}$ are moderately effective in identifying SGA newborns with abnormal structural MRI (Figure 3A,B).

In summary, TRUST and PC MRI are useful methods for assessing cerebral oxygen consumption and hemodynamics in SGA neonates. While SGA neonates with normal structural MRI have similar physiological parameters to AGA neonates, SGA neonates with abnormal structural MRI showed reducedCMRO ${ }_{2}$ and CBF. Physiological imaging may be useful in identifying SGA neonates at high risk of developing irreversible brain damage.

\section{Limitations}

It is widely established that localized measurements of physiological parameters for localized brain injury are more sensitive than global measurements, the latter of which can lead to false-negative results in inter-group comparisons; this was a limitation of this study. Considering the CBF autoregulation, and neuroplasticity that occurs after brain injury, global measurement of physiological parameters also have reference values for diagnosis and therapy in the early stages. In the future, we will combine the methods of near infrared reflectance spectroscopy (NIRS) and neurite orientation dispersion and density imaging (NODDI) to measure physiological parameters and microstructural alteration in different areas (28), which might produce new findings.

\section{Acknowledgments}

Funding: This study was supported by the Science and Technology Planning Project of Shenyang (No. 19-1124-013), the National Natural Science Foundation of China (Grant Agreement No. 81871408), the Medical Education Science Research of China Medical University (No. YDJK2020042), and the New Technology Project of Shengjing Hospital.

\section{Footnote}

Conflicts of Interest: All authors have completed the ICMJE uniform disclosure form (available at http://dx.doi. org/10.21037/qims-20-1040). The authors have no conflicts of interest to declare.

Ethical Statement: The Ethics Committee approved the study protocol of Shengjing Hospital of China Medical University (IRB2015PS28K). Written informed consent was waived on the basis that the data were collected as part 
of a clinically indicated MRI.

Open Access Statement: This is an Open Access article distributed in accordance with the Creative Commons Attribution-NonCommercial-NoDerivs 4.0 International License (CC BY-NC-ND 4.0), which permits the noncommercial replication and distribution of the article with the strict proviso that no changes or edits are made and the original work is properly cited (including links to both the formal publication through the relevant DOI and the license). See: https://creativecommons.org/licenses/by-nc-nd/4.0/.

\section{References}

1. Sharma D, Shastri S, Sharma P. Intrauterine Growth Restriction: Antenatal and Postnatal Aspects. Clin Med Insights Pediatr 2016;10:67-83.

2. Ding G, Tian Y, Zhang Y, Pang Y, Zhang JS, Zhang J. Application of a global reference for fetal-weight and birthweight percentiles in predicting infant mortality. BJOG 2013;120:1613-21.

3. Christian P. Fetal growth restriction and preterm as determinants of child growth in the first two years and potential interventions. Nestle Nutr Inst Workshop Ser 2014;78:81-91.

4. Cheng J, Li J, Tang X. Analysis of perinatal risk factors for small-for-gestational-age and appropriate-for-gestationalage late-term infants. Exp Ther Med 2020;19:1719-24.

5. Garcia-Canadilla P, Rudenick PA, Crispi F, Cruz-Lemini M, Palau G, Camara O, Gratacos E, Bijnens BH. A Computational Model of the Fetal Circulation to Quantify Blood Redistribution in Intrauterine Growth Restriction. PLoS Comput Biol 2014;12:10:e1003667.

6. Knudsen VK, Orozova-Bekkevold IM, Mikkelsen TB, Wolff S, Olsen SF. Major dietary patterns in pregnancy and fetal growth. Eur J Clin Nutr 2008;62:463-70.

7. Niu Z, Xie C, Wen X, Tian F, Ding P, He Y, Lin J, Yuan S, Guo X, Jia D, Chen WQ. Placenta mediates the association between maternal second-hand smoke exposure during pregnancy and small for gestational age. Placenta 2015;36:876-80.

8. Estourgie-van Burk GF, Bartels M, Hoekstra RA, Polderman TJ, Delemarre-van de Waal HA, Boomsma DI. A twin study of cognitive costs of low birth weight and catch-up growth. J Pediatr 2009;154:29-32.

9. Dani KA, Thomas RG, Chappell FM, Shuler K, Muir KW, Wardlaw JM. Systematic review of perfusion imaging with computed tomography and magnetic resonance in acute ischemic stroke: heterogeneity of acquisition and postprocessing parameters: a translational medicine research collaboration multicentre acute stroke imaging study. Stroke 2012;43:563-6.

10. Wright EA, d'Esterre CD, Morrison LB, Cockburn N, Kovacs M, Lee TY. Absolute cerebral blood flow infarction threshold for 3-hour ischemia time determined with CT perfusion and 18F-FFMZ-PET imaging in a porcine model of cerebral ischemia. PLoS One 2016;11:e0158157.

11. Hashem M, Zhang Q, Wu Y, Johnson TW, Dunn JF. Using a multimodal near-infrared spectroscopy and MRI to quantify gray matter metabolic rate for oxygen: A hypothermia validation study. Neuroimage 2020;206:116315.

12. Kusaka T, Isobe K, Yasuda S, Koyano K, Nakamura S, Nakamura M, Ueno M, Miki T, Itoh S. Evaluation of cerebral circulation and oxygen metabolism in infants using near-infrared light. Brain Dev2014;36:277-83.

13. Colditz P, Greisen G, Pryds O. Comparison of electrical impedance and 133xenon clearance for the assessment of cerebral blood flow in the newborn infant. Pediatr Res 1988;24:461-4.

14. Liu P, Huang H, Rollins N, Chalak LF, Jeon T, Halovanic C, Lu H. Quantitative assessment of global cerebral metabolic rate of oxygen (CMRO2) in neonates using MRI. NMR Biomed 2014;27:332-40.

15. Qi Y, Liu P, Lin Z, Lu H, Wang X. Hemodynamic and Metabolic Assessment of Neonates With Punctate White Matter Lesions Using Phase-Contrast MRI and T2Relaxation-Under- Spin-Tagging (TRUST) MRI. Front Physiol 2018;9:233.

16. Shetty AN, Lucke AM, Liu P, Sanz Cortes M, Hagan JL, Chu ZD, Hunter JV, Lu H, Lee W, Kaiser JR. Cerebral oxygen metabolism during and after therapeutic hypothermia in neonatal hypoxic-ischemic encephalopathy: a feasibility study using magnetic resonance imaging. Pediatr Radiol 2019;49:224-33.

17. De Vis JB, Petersen ET, Alderliesten T, Groenendaal F, de Vries LS, van Bel F, Benders MJ, Hendrikse J. Noninvasive MRI measurements of venous oxygenation, oxygen extraction fraction and oxygen consumption in neonates. Neuroimage 2014;95:185-92.

18. Wintermark P, Hansen A, Warfield SK, Dukhovny D, Soul JS. Near-infrared spectroscopy versus magnetic resonance imaging to study brain perfusion in newborns with hypoxic-ischemic encephalopathy treated with hypothermia. Neuroimage 2014;85:287-93.

19. Baenziger O, Jaggi JL, Mueller AC, Morales CG, Lipp 
HP, Lipp AE, Duc G, Bucher HU. Cerebral blood flow in preterm infants affected by sex, mechanical ventilation, and intrauterine growth. Pediatr Neurol 1994;11:319-24.

20. Fenton TR, Kim JH. A systematic review and metaanalysis to revise the Fenton growth chart for preterm infants. BMC Pediatr 2013;13:59.

21. Silva IBD, Cunha PAGD, Linhares MBM, Martinez FE, Camelo Júnior JS. Neurobehavior of preterm, small and appropriate for gestational age newborn infants. Rev Paul Pediatr 2018;36:407-14.

22. Liu P, Chalak LF, Krishnamurthy LC, Mir I, Peng SL, Huang H, Lu H. T1 and T2 values of human neonatal blood at 3 Tesla: Dependence on hematocrit, oxygenation, and temperature. Magn Reson Med 2016;75:1730-5.

23. Herscovitch P, MintunMA, Raichle ME. Brain oxygen utilization measured with oxygen-15 radiotracers and positron emission tomography: generation of metabolic images. J Nucl Med1985;26:416-7.

24. Burgess KR, Lucas SJ E, Burgess KM, Sprecher KE,

Cite this article as: Qi Y, Wang X, Mao J. Quantitative assessment of cerebral metabolism and hemodynamics in smallfor-gestational-age (SGA) newborns. Quant Imaging Med Surg 2021;11(6):2321-2332. doi: 10.21037/qims-20-1040
Donnelly J, Basnet AS, Tymko MM, Day T, Smith K, Lewis N, Ainslie PN. Increasing cerebral blood flow reduces the severity of central sleep apnea at high altitude. J Appl Physiol (1985) 2018;124:1341-8.

25. Liu P, Xu F, Lu H. Test-retest reproducibility of a rapid method to measure brain oxygen metabolism. Magn Reson Med 2013;69:675-81.

26. Guyton AC, Hall JE. Respiration. In: Guyton AC, Hall JE. editors. Textbook of Medical Physiology. Saunders/ Elsevier: Philadelphia, PA; 2005;502-13.

27. Peng SL, Dumas JA, Park DC, Liu P, Filbey FM, McAdams CJ, Pinkham AE, Adinoff B, Zhang R, Lu $\mathrm{H}$. Age-related increase of resting metabolic rate in the human brain. Neuroimage 2014;98:176-83.

28. Li CX, Patel S, Zhang X. Evaluation of multi-shell diffusion MRI acquisition strategy on quantitative analysis using multi-compartment models. Quant Imaging Med Surg 2020;10:824-34. 